Journal of Clinical and Nursing Research

Research Article

\title{
Endovascular Application of Magnetic Resonance Double Mismatch Technique for Acute Anterior Circulation Large Vessel Occlusion with Cerebral Infarction in an Unknown Time Window
}

\author{
Xiangkong Song ${ }^{1}$, Qing Zhang ${ }^{2}$, Lilin Gao ${ }^{1}, \mathrm{Jie} \mathrm{Q}^{3}$, Guoqing Wang ${ }^{1 *}$ \\ ${ }^{1}$ Department of Neurology, Binzhou People's Hospital, Binzhou 256600, Shandong Province, China; \\ ${ }^{2}$ Department of Neurosurgery, Binzhou People's Hospital, Binzhou 256600, Shandong Province, China \\ ${ }^{3}$ Department of Gerontology, Binzhou Medical University Hospital, Binzhou 256603, Shandong Province, China
}

\begin{abstract}
[Abstract] Objective: To investigate the clinical effects of applying the magnetic resonance double mismatch technique to endovascular treatment of acute anterior circulation, large vessel occlusion with cerebral infarction in an unknown time window. Methods: The research work was carried out in our hospital, the work was carried out from November 2018 to November 2019 , the patients with acute anterior circulation large vessel occlusion with cerebral infarction who were treated in our hospital during this period, 100 patients, 50 patients with an unknown time window and 50 patients with definite time window were selected, and they were named as the experimental and control groups, given different examination methods, were given to investigate the clinical treatment effect. Results: Patients' data on HIHSS score before treatment, the incidence of intracranial hemorrhage and rate of Mrs $\leq 2$ rating after 90 days of treatment were not significantly different $(\mathrm{P}>0.05)$, which was not meaningful. The differences in data between the two groups concerning HIHSS scores were relatively significant before, and after treatment $(P<0.05)$. Conclusion: The magnetic resonance double mismatch technique will be applied in the endovascular treatment of acute anterior circulation large vessel occlusion with cerebral infarction of unknown time window.
\end{abstract}

Keywords: Magnetic resonance double mismatch technique; Unknown time window; Acute anterior circulation large vessel occlusion with cerebral infarction; Therapeutic effect
Publication date: September, 2020

Publication online: 30 September, 2020

*Corresponding author: Guoqing Wang, wgqbrm@ sina.com

According to the current clinical development, it is highly effective and safe to treat patients with cerebral infarction associated with acute anterior circulation large vessel occlusion by applying the endovascular mechanical embolization method. However, some of the patients have unknown onset time window, so it is difficult to determine the treatment of such brain infarction patients conclusively, so it is necessary to start from the current domestic and foreign guidelines recommendations and exclude them through intravenous thrombolysis or endovascular treatment ${ }^{[1]}$. Therefore, the efficient and accurate evaluation of patients and the selection of appropriate treatment methods are essential to ensure patients' health and treatment effect ${ }^{[2]}$. In this study, the magnetic resonance double mismatch technique is applied to investigate the impact of clinical intervention.

\section{Patient data and study methods}

\subsection{General information}

Take our patients as an example, 100 vascular patients with acute anterior circulation large vessel occlusion with cerebral infarction in an unknown time window 
were selected, the study was carried out from November 2018 to November 2019, the patients were grouped according to their actual conditions, one group was the unknown time window, named the experimental group, the number was 50, 34 of the patients were male, and 16 were female, the age of the patients was selected in the range of 60 years- 87 years, the mean age of the patients was $(62.35 \pm 434)$ years. The other group was given for the bright time window, named control group, the number of 50, including the number of men and women were 35 and 15 , respectively, the age selection range was $62-88$ years, the mean age was $(63.04 \pm 4.05)$ years. The general information of the two groups of patients were not significantly different, $\mathrm{P}>0.05$, which could be compared. This study was approved by the ethics committee of the hospital, and the patients all agreed and signed the consent form, which could exclude the possibility that the patients had a psychiatric history and other significant diseases.

\subsection{Research methodology}

Examination equipment and methods: A GE Discovery MR 750 3.0T scanner is applied to implement diffusionweighted imaging techniques and fluid attenuation inversion recovery sequences, and the patient is given a 3D arterial spin labelling examination and magnetic resonance angiography. Patients were helped to select a supine position, the patient's head was placed in the coil, and the patient was subsequently repositioned to a standard horizontal position, with a baseline of the auditory angular line for scanning the whole brain.

Image and processing analysis: The raw data of 3DASL images were all transferred to ADW4.6 workstation to give its data processing, and the arterial spin cerebral blood flow map was acquired by Func-tool software, in which the pseudo-colour map colour is blue to red transition. Two radiologists and a neurologist were selected for analysis to determine the extent of new infarction in their hypoperfused areas and DWI, and the relative cerebral blood flow between the field of new infarction and the contralateral healthy tissue was calculated. The MRA test was refined to determine the macrovascular condition of the lesion area.

Reperfusion therapy: The criteria for reperfusion therapy for acute anterior circulation large vessel occlusion with cerebral infarction in an unspecified time window include a maximum area of 1.2 or higher in the hypoperfused zone of 3DASL and a combination of large vessel occlusion and failure to visualize the DWI high signal zone of FLAIR. Patients with unknown time window need to be given intravenous thrombolysis and endovascular therapy. Patients with a precise time window, on the other hand, need to be given intravenous thrombolysis followed by endovascular treatment. In the implementation of the method of intravenous thrombolysis: aprotinin was applied, mainly with the drug produced by Boehringer Ingelheim, Germany, at a dose of $50 \mathrm{mg} / \mathrm{branch}$. Each application of $0.6 \mathrm{mg} /$ $\mathrm{kg}$ requires the patient to be given an intravenous push at a concentration of $10 \%$. In contrast, the remaining dose is given a continuous intravenous pump within an hour. For the endovascular approach: patients are given stents for embolization, and after contact aspiration of the intermediate catheter, they also need to be given angioplasty interventions if they have heavy residual stenosis $^{[3]}$.

\subsection{Statistical methods}

The statistical software SPSS20.0 was used as a tool to analyze the data presented in this study statistically. The comparison results of the measurement data $(\mathrm{x} \pm \mathrm{s})$ were verified by t-values, and the comparison results of the count data $(\mathrm{n}, \%)$ were confirmed by 2 -values ${ }^{[4]}$.

\section{Results}

\subsection{Effectiveness of thromboprophylaxis}

The differences between the two groups in terms of HIHSS score, the incidence of intracranial hemorrhage and rate of $\mathrm{Mrs} \leq 2$ rating after 90 days of treatment were not significant, $\mathrm{P}>0.05$, which is not meaningful, but in comparison, the differences between the two groups in terms of HIHSS score before and after treatment were relatively large, $\mathrm{P}<0.05$, which is meaningful.

Table 1. Comparison of thrombosis treatment in the

\begin{tabular}{|c|c|c|c|c|}
\hline Grouping & $\begin{array}{l}\text { Pre-treatment } \\
\text { HIHSS score }\end{array}$ & $\begin{array}{l}\text { Post-treatment } \\
\text { HIHSS score }\end{array}$ & $\begin{array}{l}\text { Intracranial } \\
\text { haemorrhage } \\
(\%)\end{array}$ & $\begin{array}{l}90 \text { days of } \\
\text { treatment Mrs } \\
\leq 2 \text { points } \\
(\%)\end{array}$ \\
\hline $\begin{array}{l}\text { Pilot } \\
\text { group } \\
\qquad(n=50)\end{array}$ & $18.44 \pm 4.34$ & $7.64 \pm 4.24$ & $7(14.00)$ & $25(50.00)$ \\
\hline $\begin{array}{l}\text { Control } \\
\text { group } \\
\qquad(n=50)\end{array}$ & $16.04 \pm 5.45$ & $6.05 \pm 5.44$ & $8(16.00)$ & $26(52.00)$ \\
\hline$X^{2} / t$ & 0.480 & 0.496 & 0.045 & 0.088 \\
\hline $\mathrm{P}$ & $>0.05$ & $>0.05$ & $>0.05$ & $>0.05$ \\
\hline
\end{tabular}




\subsection{Case-specific analysis}

The patient is a male, age 63, and the patient mainly has lateral weakness problems, 6 hours after the onset of symptoms, the patient was admitted to the hospital, given an NIHSS score of 10, the patient's MRI has 3DASL-DWI, FLAIR-DWI double mismatch, the patient's MRA suggests that the patient's left middle cerebral artery M1 distal occlusion, given its stent to retrieve the embolism, and conducted The TICI grade, a 3, was 0 on the NIHSS score and 0 on the 90 -day mRS score after giving the patient a 7-day therapeutic intervention. The perfusion of their 3DASL was significantly improved when they reviewed the MRA for vascular patency of the original lesion.

\section{Discussion}

With the advancement of medical technology in China, studies such as MR-CLEAN, ESCAPE, SWIFT PRIME, REVASCAT, and EXTEND-IA have emerged, laying the evidence base for the recommendation of embolization within 6 hours of onset in patients with anterior circulation ischemic stroke, followed by its prospective 6-16 hour time window, and unfolded a multicenter MRI and perfusion chancellor techniques have been used to prospectively develop studies for the time window, mainly analyzing patients with stroke with an unmatched clinical image over the time window, and to achieve the practice of embolization of stroke patients through the management of the tissue window to ensure better treatment for patients ${ }^{[5]}$. However, there are still $30 \%$ of patients with acute cerebral infarction of unknown time window in which the reperfusion therapy manifests a global problem. According to the results of relevant research data, these patients with acute cerebral infarction of strange onset time have similar clinical features and imaging manifestations as patients with a precise onset time of 3-6 hours, which can be derived to evaluate the pathophysiological condition of the patients in line with the results ${ }^{[6]}$. In the case of awakened stroke patients, which usually occurs before the patient awakens, the possibility of reperfusion therapy exists in such patients.

The therapeutic time window is critical, but in patients with an unknown time window, it's even more essential to individualize the evaluation of the pathophysiological condition of patients with ischemic brain tissue. For acute coronary, cerebrovascular disease, its DWI high signal mainly indicates a state of restricted Brownian motion of water molecules in the presence of toxic edema of cells, which can be developed by the patient several minutes after the infarction ${ }^{[7]}$. However, FLAIR is more sensitive to the problem of vasogenic edema, which manifests itself within a few hours after the infarction, and the presence of a partial FAIR-DWI mismatch in awakened stroke patients. Some researchers have suggested that patients with this mismatch can be treated with reperfusion therapy. In the case of the WAKE-UP study, which used the mismatch between DWI and FLAIR to guide intravenous thrombolysis for an unknown time window, the corresponding 90-day functional outcome was more pronounced when compared with the placebo group $^{[8]}$. MR WITNESS is another study based on the mismatch between DWI and FLAIR to select patients with relative ischemic stroke for intravenous thrombolysis, and the results were similar to the previous one. However, neither of these studies was genuinely realized for the ischemic semi-dark band ${ }^{[9]}$. Magnetic resonance perfusion-weighted imaging can respond to coronary hypoperfusion extent, which is a commonly used method for IP determination in clinical practice.3DASL, on the other hand, is acquired from the helical K-space of the fast spin-echo, which can overcome motion artifacts and magnetically sensitive artifacts cases introduced by planar echo imaging. The quality of the impact is better and allows for rapid acquisition while corresponding to a broader imaging range with more stable signal localization, which has been widely used in the clinical setting ${ }^{[10]}$.

In summary, magnetic resonance double mismatch technique has been applied to the endovascular treatment of acute anterior circulation large vessel occlusion with cerebral infarction in the unknown time window.

\section{References}

[1] Huang WL, Gong SJ, Wu ZS, et al. Clinical outcome analysis of bridging therapy and mechanical thrombosis in patients with acute cerebral infarction and anterior circulation large vessel occlusion[J]. Journal of Stroke and Neurological Disorders, 2019, 36(2): 147-149.

[2] Wen HF, Wang RT, Li JL, et al. Endovascular treatment of patients with acute anterior circulation large vessel occlusion with cerebral infarction of unknown time window with magnetic resonance double mismatch technique[J]. Chinese Journal of Geriatric Cardiovascular Disease, 2019, 21(6): 568-571.

[3] Chinese Journal of Geriatric Cardiovascular and Cerebrovascular Diseases, Index of Subject Words[J]. Chinese Journal of Geriatric Cardiovascular and Cerebrovascular Diseases, 2019, 21(12): 1345-1360. 
[4] Cheng L, Li XD, Wang SB, et al. Endovascular treatment of acute ischemic stroke due to anterior circulation large vessel occlusion associated with atherosclerosis[J]. Lingnan Modern Clinical Surgery, 2020, 20(1): 98-101+107.

[5] Yang D. A preliminary study of endovascular treatment options for acute anterior circulation large vessel occlusion[D]. Second Military Medical University, 2017.

[6] Yu M. Correlation analysis between acute ischemic stroke anterior circulation large vessel occlusion and NIHSS score [D]. Jianghan University, 2019.

[7] Shang XJ. Feasibility analysis of endovascular treatment in patients with mild ischemic stroke and anterior circulation large vessel occlusion[D]. Nanjing Medical University, 2019.

[8] Liu GL. Comparison of the efficacy of intravenous thrombolysis and intravenous thrombolysis bridging endovascular treatment for acute large vessel occlusive stroke [D]. Yanbian University,2019.

[9] Yao WT. Clinical study of contrast dye staining of brain tissue after mechanical thrombectomy for acute ischemic stroke[D]. Hebei Medical University, 2018.

[10]Zuo M. Effect of stroke staging on the prognosis of endovascular treatment of acute anterior circulation massive vessel occlusion stroke[D]. Southeast University, 2018. 